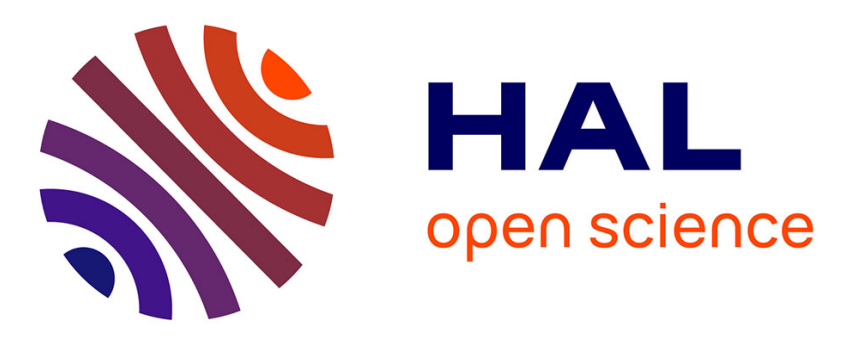

\title{
Estimates for localised transverse electric modes in multi-structured crystal fibres
}

Natasha Movchan, Sébastien Guenneau, Alexander Movchan, Ross Mcphedran

\section{To cite this version:}

Natasha Movchan, Sébastien Guenneau, Alexander Movchan, Ross Mcphedran. Estimates for localised transverse electric modes in multi-structured crystal fibres. Physica B: Condensed Matter, 2007, 394 (2), pp.281-284. hal-00186680

\section{HAL Id: hal-00186680 https://hal.science/hal-00186680}

Submitted on 3 Dec 2007

HAL is a multi-disciplinary open access archive for the deposit and dissemination of scientific research documents, whether they are published or not. The documents may come from teaching and research institutions in France or abroad, or from public or private research centers.
L'archive ouverte pluridisciplinaire HAL, est destinée au dépôt et à la diffusion de documents scientifiques de niveau recherche, publiés ou non, émanant des établissements d'enseignement et de recherche français ou étrangers, des laboratoires publics ou privés. 


\title{
Estimates for localised transverse electric modes in multi-structured crystal fibres
}

\author{
N.V. Movchan ${ }^{\text {a }}$ S. Guenneau ${ }^{\mathrm{a}, \mathrm{b}}$ A.B. Movchan ${ }^{\mathrm{a}}$ R.C. McPhedran ${ }^{\mathrm{c}}$ \\ ${ }^{a}$ Department of Mathematical Sciences, $M \& \mathcal{O}$ Building, \\ Peach Street, Liverpool L69 3BX, United Kingdom \\ ${ }^{\mathrm{b}}$ Institut Fresnel, UMR CNRS 6133, University Aix-Marseille III, case 162, F13397 Marseille Cedex 20, France \\ ${ }^{\mathrm{c}}$ CUDOS ARC Centre of Excellence, University of Sydney, 2006, Australia.
}

\begin{abstract}
The paper presents analytical and numerical models describing localised transverse electric defect modes in a doubly periodic structure involving split ring inclusions. We provide an asymptotic estimate which can be implemented in the optimal design of low frequency stop bands in photonic crystal fibres.
\end{abstract}

Key words: Thin-bridges, split ring resonators, optical fibres PACS: 47.11.St, 42.15.-i, 78.20.Bh

\section{Introduction}

Split ring resonators (SRR) have recently attracted a great deal of attention since they are building blocks of socalled meta-materials exhibiting artificial magnetism [1]. Here, we propose to use them as the building blocks of locally resonant photonic crystal fibres. The idea is to replace each of the six air holes of the micro-structured fibre studied in [2] by a SRR. The dispersion diagrams for electromagnetic waves in such a structure possess low frequency stop bands. The boundaries of these stop bands can be evaluated analytically and hence the filtering properties can be controlled by changing the physical parameters of the system.

\section{Formulation of the problem}

We consider transverse electric waves propagating within a doubly periodic array of 'defects' represented by a 'cloud' of 6 voids $\Omega_{1}, \Omega_{2}, \ldots, \Omega_{6}$ embedded in an hexagonal elementary cell $Y$ (see figure 1(a)). In this case, the time harmonic transverse electric field has the form $\mathbf{E}(x, y, t)=$ $\left(E_{1}(x, y), E_{2}(x, y), 0\right) e^{-i \omega t}$ so that its magnetic counterpart $\mathbf{H}(x, y, t)=\left(0,0, H_{3}(x, y)\right) e^{-i \omega t}$ is longitudinal. The propagation of these waves satisfies the Helmholtz equation taken in distributional sense

$$
\left(\nabla \cdot \frac{1}{n^{2}(x, y)} \nabla\right) H_{3}(x, y)+\frac{\omega^{2}}{c^{2}} H_{3}(x, y)=0
$$

where $c$ is the speed of light in vacuum, $\omega$ the wave frequency and $n$ the refractive index such that $\alpha \geq n \geq \beta>0$, where $\alpha$ and $\beta$ are positive arbitrary constants. We look for solutions $H_{3}$ of finite energy in $Y$ which satisfy the FloquetBloch condition

$H_{3}\left(\left(x+p \mathbf{l}^{(1)}, y+q \mathbf{l}^{(2)}\right)=H_{3}(x, y) e^{i\left(k_{1} p+k_{2} q\right)}\right.$,

where $\mathbf{l}^{(1)}=d(1,0), \mathbf{l}^{(2)}=d / 2(1, \sqrt{3})$ are the lattice vectors, $d$ is the period of the structure, $p$ and $q$ are integers, and $\mathbf{k}=\left(k_{1}, k_{2}\right)$ represents the Bloch vector.

Equation (1) is then solved numerically by considering its weak formulation using test functions $V$ taking values on nodes of a triangular mesh of the basic cell $Y$. The Finite element formulation was used in the numerical computations with Getdp [3] where special care was taken to enforce Floquet-Bloch conditions [4].

\section{Helmholtz equation within thin-bridges}

Here, the metallic inclusions $\Omega_{1}, \ldots, \Omega_{6}$ are of the shape of the letter $C$ and they are located and oriented in such a way as to preserve the six-fold symmetry (figure 1). Formally, using local coordinates associated with each inclusion

$\Omega_{j}=\left\{a_{j}<\sqrt{x^{2}+y^{2}}<b_{j}\right\} \backslash \overline{\Pi_{\varepsilon}^{(j)}}, j=1, \ldots, 6$, 

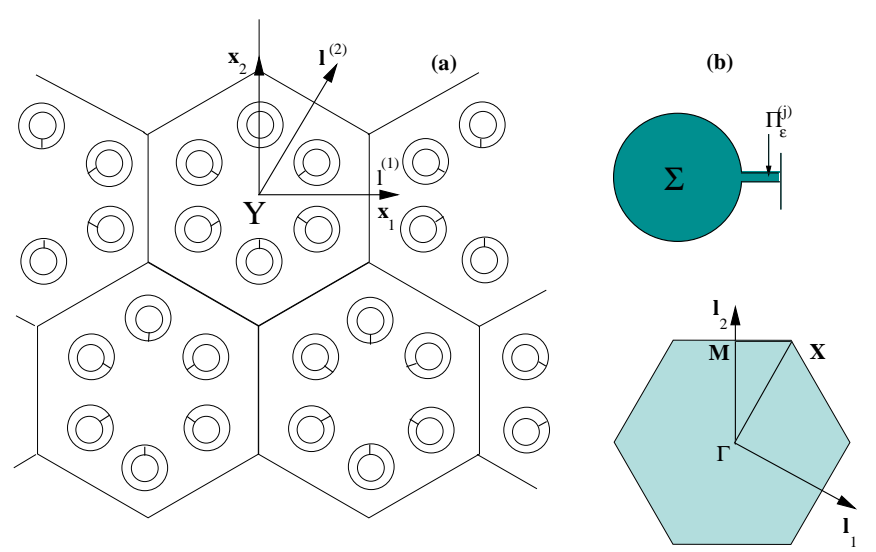

(c)

Fig. 1. (a) Physical space. A doubly periodic array of hexagonal macro-cells containing six split ring resonators located and oriented to preserve six-fold symmetry. b) Associated multi-structure consisting of a large body $\Sigma$ connected to a thin bridge $\Pi_{\varepsilon}^{(j)}$ (c) Reciprocal space. Irreducible part of the first Brillouin zone $\Gamma M X$ where $\Gamma=(0,0), M=(0,2 \pi /(\sqrt{3} d))$ and $X=(2 \pi /(3 d), 2 \pi /(\sqrt{3} d))$.

where $a_{j}$ and $b_{j}$ are given constants, and

$\Pi_{\varepsilon}^{(j)}=\left\{(x, y): a_{j}<x<b_{j},|y|<\varepsilon h_{j} / 2\right\}$,

is a thin ligament between the 'ends of the letter $C$ '. Here $\varepsilon h_{j}$ is the thickness of the $j$-th bridge, with $\varepsilon$ being a small positive non-dimensional parameter.

The function $H_{3}(x, y)$ satisfies the Helmholtz equation within the thin-ligament $\Pi_{\varepsilon}^{(j)}$ occupied by a material of constant refractive index $n_{j}$,

$\nabla^{2} H_{3}(x, y)+n_{j}^{2} \frac{\omega^{2}}{c^{2}} H_{3}(x, y)=0$

together with homogeneous Neumann boundary conditions (infinite conducting walls)

$\left.\frac{\partial H_{3}}{\partial y}\right|_{y= \pm \varepsilon h_{j} / 2}=0$.

This system will be supplied with appropriate boundary conditions at the ends $x=a_{j}$ and $x=b_{j}$. From now on, we drop the index $j$ for simplicity.

\section{Thin-bridge analysis}

Let us introduce the scaled variable $\xi=\frac{y}{\varepsilon}$. Hence, $\frac{\partial^{2} v}{\partial y^{2}}=$ $\frac{1}{\varepsilon^{2}} \frac{\partial^{2} v}{\partial \xi^{2}}$, and the rescaled wave equation in $\Pi_{1}=\{(x, \xi)$ : $a<x<b,|\xi|<h / 2\}$ becomes

$\left(\frac{1}{\varepsilon^{2}} \frac{\partial^{2}}{\partial \xi^{2}}+\frac{\partial^{2}}{\partial x^{2}}+n^{2} \frac{\omega^{2}}{c^{2}}\right) H_{3}=0$.

The field $H_{3}$ is approximated in the form

$H_{3} \sim H_{3}^{(0)}(x, \xi)+\varepsilon^{2} H_{3}^{(1)}(x, \xi)$.
To the leading order we obtain (see (6), (7) and (8))

$\frac{\partial^{2} H_{3}^{(0)}}{\partial \xi^{2}}=0,|\xi|<h / 2,\left.\frac{\partial H_{3}^{(0)}}{\partial \xi}\right|_{\xi= \pm h / 2}=0$.

Hence, $H_{3}^{(0)}=H_{3}^{(0)}(x)$ (it is $\xi$-independent). The function $H_{3}^{(1)}$ satisfies the following model problem on the scaled cross-section of $\Pi_{1}$

$$
\begin{gathered}
\frac{\partial^{2} H_{3}^{(1)}}{\partial \xi^{2}}=-\frac{\partial^{2} H_{3}^{(0)}}{\partial x^{2}}-n^{2} \frac{\omega^{2}}{c^{2}} H_{3}^{(0)} \quad,|\xi|<h / 2, \\
\frac{\partial H_{3}^{(1)}}{\partial \xi} \mid \xi= \pm h / 2 \\
\mid \xi=0 .
\end{gathered}
$$

The solvability condition for the problem (10) gives us the equation for $H_{3}^{(0)}$, that is,

$\frac{d^{2} H_{3}^{(0)}}{d x^{2}}+n^{2} \frac{\omega^{2}}{c^{2}} H_{3}^{(0)}=0, a<x<b$.

Thus, to the leading order, we can approximate the field $H_{3}$ within the thin bridge $\Pi_{\varepsilon}$ by the function $H_{3}^{(0)}(x)$ which satisfies the one-dimensional Helmholtz equation.

\section{An asymptotic estimate of the frequency of the localised mode}

We are interested in standing waves (flat curves on the dispersion diagram of Figure 2). For low frequencies, let $C_{1}$ denote the constant value of the field in the core $\Sigma=$ $\left\{\sqrt{x^{2}+y^{2}}<b\right\}$ of the multi-structure $\Omega$, and $C_{2}$ denote the constant value of the field within the complementary area of the macro-cell $Y \backslash \Omega$ excluding the ligaments. Then, neglecting the small area of the ligament, we obtain

$C_{1} \mathcal{S}_{\Sigma}+C_{2} \mathcal{S}_{Y \backslash \Omega}=O(\varepsilon)$

where $\mathcal{S}_{\Sigma}$ and $\mathcal{S}_{Y \backslash \Omega}$ denote the areas of $\Sigma$ and $Y \backslash \Omega$.

Boundary layers occur at the end regions of the thin ligaments [6]. These boundary layers are characterized by exponential decay when $H_{3}^{(0)}$ satisfies:

$H_{3}^{(0)}(a)=C_{1}$

$H_{3}^{(0)}(b)=C_{2}=-\frac{\mathcal{S}_{\Sigma}}{\mathcal{S}_{Y \backslash \Omega}} C_{1}$.

Now, integrating (5) over the multistructure $\Omega$ and applying the divergence theorem, we obtain that to order $\varepsilon$

$\left.\frac{d H_{3}^{(0)}}{d x}\right|_{x=a}=-\frac{n^{2} \mathcal{S}_{\Sigma} \omega^{2}}{c^{2} \varepsilon h} C_{1}$.

Combining (11), (13) and (14), in the low frequency limit we obtain

$\omega^{2} \sim \frac{\varepsilon h c^{2}}{(b-a) \mathcal{S}_{\Sigma} n^{2}}\left(1+\frac{\mathcal{S}_{\Sigma}}{\mathcal{S}_{Y \backslash \Omega}}\right)$.

We note that this estimate is in agreement with the one derived in [5] for anti-plane shear waves when $\mathcal{S}_{\Sigma} / \mathcal{S}_{Y \backslash \Omega}$ vanishes. 


\section{Thin-bridge estimates versus finite elements results}

Formula (15) is an accurate estimate for the frequency of TE modes trapped in split ring resonators. Here $n$ is the refractive index of the dielectric material within the SRR, $\varepsilon h$ is the constant thickness of the thin bridge (split), $\mathcal{S}_{\Sigma}$ is the area of the core of the ring, and $\mathcal{S}_{Y \backslash \Omega}$ is the area of the hexagonal cell excluding the six rings and their cores.

In our case, $\varepsilon h=0.012, \mathcal{S}_{\Sigma}=\pi * 0.159^{2}, b-a=0.1$ and $n=\sqrt{12}$, and $\mathcal{S}_{Y \backslash \Omega}=3 \sqrt{3} / 2-6 \pi 0.259^{2}=3.93$, so that $\frac{\mathcal{S}_{\Sigma}}{\mathcal{S}_{Y \backslash \Omega}}=0.02$. Hence, we find $\omega_{1} d / c=0.3548$ whereas the finite element computation gives $\omega_{1}^{*} d / c=0.35308$. This is associated with the lower flat band on the right dispersion diagram of Figure 2. We notice that this first localised eigenmode is axi-symmetric (constant) within each inclusion except for thin bridges. Its analytic expression in thin bridges is given by

$$
\begin{aligned}
H_{3}^{(0)}(x) & =-\frac{C_{2} \cos (\omega(b-a) / c)-1}{\sin (\omega(b-a) / c)} \sin (\omega x / c) \\
& +C_{2} \cos (\omega x / c) .
\end{aligned}
$$

\section{Higher localised modes and possible applications}

Finally, the upper flat curve (the seventh dispersion curve) in figure 2 corresponds to a dipole mode localised within each structured inclusion. In this case, the thin bridges play little part. Hence, if we denote the refractive index of the core of the inclusion by $n$, such a mode is simply the solution of the Helmholtz equation

$\Delta H_{3}+n^{2} \frac{\omega^{2}}{c^{2}} H_{3}=0, x_{1}^{2}+x_{2}^{2}<r_{1}^{2}$.

In polar coordinates, the dipole solution of this problem is

$H_{3}(r, \theta)=J_{1}\left(\frac{\omega n r}{c}\right) e^{i \theta}$.

Further, the dipole mode we are looking for satisfies the Neumann condition $\partial H_{3} / \partial r=0$ on the inner circular boundary $r=r_{1}$ of the split ring. Hence, the associated frequency is given by the first root of the transcendental equation

$\frac{\omega n}{c} J_{1}^{\prime}\left(\frac{\omega n r_{1}}{c}\right)=0$,

where $r_{1}=0.159 d$ and $n=\sqrt{12}$. This gives an estimate $\omega_{2} d / c=3.36$ for the normalised frequency of the first dipole mode of split rings (see Figure 2(b)) which fits reasonably well the numerical value $\omega_{F E M}=\omega_{2}^{*} d / c=3.4540$ given by Finite Element Computations.

Dispersionless modes propagating along the crystal fiber at very low frequency (as it is the case for the localised mode associated with the lower flat band) may lead to subwavelength imaging in a way similar to that demonstrated in [7] for swiss-rolls at radio frequencies. Modes associated with flat curves at higher frequencies suggest that they would be nearly leakage free in the case of multi-structured fibres of finite cross-section. Multi-structured crystal fibres hence have possible applications both in medical imaging (radio frequencies) and long-haul communications (from near infra-red to optical frequencies).

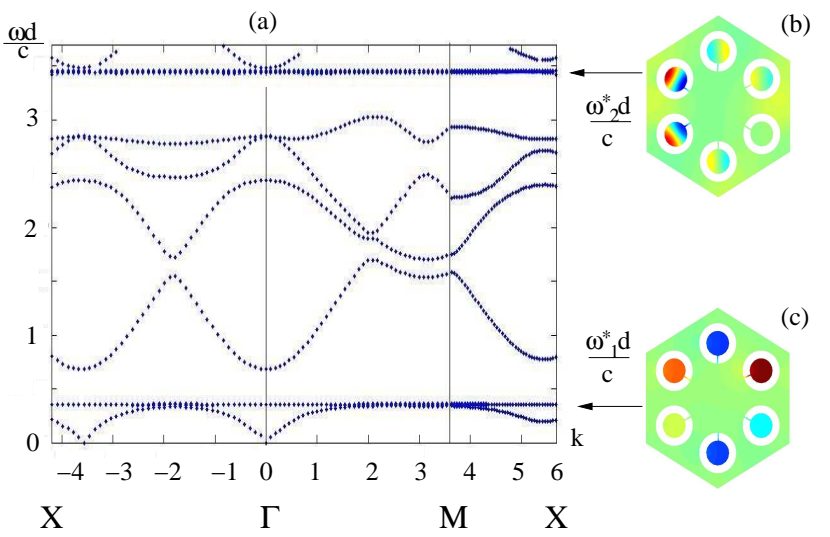

Fig. 2. Transverse incidence. (a) Dispersion diagram for six infinitely conducting inclusions shaped as split ring resonators of inner radius $0.159 d$ and outer radius $0.259 d$ (right) and filled with a dielectric material of relative permittivity 12 . The center-to-center spacing of inclusions is $0.7 d$. Inclusions are surrounded by a matrix of relative permittivity 1 . The horizontal axis shows the magnitude of the Bloch vector describing the first Brillouin zone described in Figure 1 (c). On the vertical axis, we represent the eigenfrequencies of propagating TE waves. (b) Eigenfield associated with eigenfrequency $\omega_{1}^{*} d / c=0.35308$ (root of multiplicity six) which is constant in the center of each C-shaped inclusion. (c) Eigenfield associated with eigenfrequency $\omega_{2}^{*} d / c=3.4540$ (multiple root). We note the dipole nature of modes confined in the center of each $\mathrm{C}$-shaped inclusion.

\section{Acknowledgements:}

RCM acknowledges support of London Mathematical Society under grant 4424 and the Australian Research Council through its Centre of Excellence Programme. ABM, NVM and SG acknowledge support of the Franco-British partnership programme ALLIANCE (project PN 05.026). SG acknowledges support of the EC funded project PHOREMOST under grant FP6/2003/IST/2-511616.

\section{References}

[1] Pendry, J.B. and Holden, A.J. and Stewart, W.J. and Youngs, I. IEEE Trans. Micr. Theory and Techniques 47(1999) 2075.

[2] B.T. Kuhlmey, T.P. White, R.C. McPhedran, D. Maystre, G. Renversez, C. Martin de sterke and L.C. Botten, J. Opt. Soc. Am. B 19(2002) 2331.

[3] P. Dular, C. Geuzaine, F. Henrotte and W. Legros, IEEE Trans. Mag. 34 (1998) 3395.

[4] A. Nicolet, S. Guenneau, C. Geuzaine, and F. Zolla., J. Comput. Appl. Math. 168 (2004) 321.

[5] A.B. Movchan and S. Guenneau, Phys. Rev. B 70(2004) 125116.

[6] A.B. Movchan, N.V. Movchan, Mathematical modelling of solids with non-regular boundaries, CRC Press (1995)

[7] M. Wiltshire, J.B. Pendry, I.R. Young, D.J. Larkman, D.J. Gilderdale, J.V. Hajnal Science291(2001)849. 